\title{
Differential expression and function of the endogenous lactate receptor, GPR81, in ER $\alpha$ - positive/HER2-positive epithelial vs. post-EMT triple-negative mesenchymal breast cancer cells
}

\author{
Denisse Tafur ${ }^{1}$, Patrick Svrcek $^{2}$, Bruce White ${ }^{1}$ \\ 'Department of Cell Biology, University of Connecticut Health, Farmington, CT 06032, USA. \\ ${ }^{2}$ University of Connecticut School of Medicine, University of Connecticut Health, Farmington, CT 06032, USA. \\ Correspondence to: Dr. Denisse Tafur, the Jackson Laboratory for Genomic Medicine, Farmington, CT 06032, USA. \\ E-mail: denisse.tafur@jax.org \\ How to cite this article: Tafur D, Svrcek P, White B. Differential expression and function of the endogenous lactate receptor, \\ GPR81, in ER $\alpha$-positive/HER2-positive epithelial vs. post-EMT triple-negative mesenchymal breast cancer cells. \\ J Cancer Metastasis Treat2019;5:46. http://dx.doi.org/10.20517/2394-4722.2018.102
}

Received: 20 Dec 2018 First Decision: 11 Feb 2019 Revised: 26 Mar 2019 Accepted: 5 May 2019 Published: 6 Jun 2019

Science Editor: Rafat A. Siddiqui Copy Editor: Cai-Hong Wang Production Editor: Jing Yu

\begin{abstract}
Aim: Lactate can signal through the endogenous lactate receptor, GPR81, which is expressed in some cancers. Lactate metabolism is altered by the metastasis-promoting process of epithelial-mesenchymal transition (EMT). This study examined the expression and function of GPR81 in breast cancer samples, and in receptor-positive epithelial $v s$. triple-negative post-EMT mesenchymal breast cancer cells.

Methods: GPR81 mRNA expression was examined by breast cancer microarray, and by a Kaplan-Meier survival curve. Using 3-dimensional culture conditions, GPR81 mRNA expression in epithelial and mesenchymal breast cancer cell lines was measured by qRT-PCR. GPR81 siRNA was used to assess the role of GPR81, alone or in conjunction with tamoxifen, in the regulation of MCT1 and MCT4 lactate transporters, intracellular lactate, and cell proliferation and survival.
\end{abstract}

Results: GPR81 mRNA levels were elevated in receptor-positive breast cancer, relative to non-tumor and triplenegative samples, and correlated with increased survival. GPR81 expression was elevated in the two epithelial breast cancer cell lines vs. the corresponding post-EMT mesenchymal cell lines. GPR81 knock-down in epithelial MCF7 cells caused: 1, selectively lower mRNA and protein expression of the MCT1 transporter, but not MCT2 or MCT4

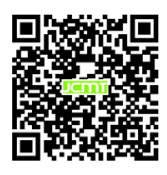


transporters; 2, lower levels of intracellular lactate; and 3, decreased proliferation and survival in lactate onlycontaining conditions. GPR81 siRNA plus tamoxifen displayed additive suppressive effects on MCT1 expression and cell viability.

Conclusion: GPR81 promotes the ability of epithelial breast cancer cells to import lactate for energy use. As such, GPR81 represents a potential target for treatment of hormone-positive breast cancer cells, and may be prognostic for higher grade breast cancer.

Keywords: GPR81, lactate metabolism, MCT transporters, MCT1, tamoxifen

\section{INTRODUCTION}

Invasive breast cancer (IBC) is the most predominant form of cancer among women worldwide and the second leading cause of cancer-related deaths in women in the United States ${ }^{[1,2]}$. Moreover, breast cancer is a complex and heterogeneous disease, which includes a range of pathological subtypes associated with different clinical behavior, treatment options and outcomes. The most common subtype of IBC, termed Luminal A, is characterized by an adhesive epithelial phenotype and defined by the overexpression of the estrogen receptor $\alpha(E R \alpha)$ that underlies the endocrine-based treatment of this subtype ${ }^{[3]}$. Although Luminal A IBC is a relatively non-aggressive type with a good response to adjuvant endocrine therapy and a high survival rate, failure of early detection is associated with metastatic disease, which in turn is responsible for the majority of deaths in breast cancer patients.

The metastatic dissemination of epithelial IBC cells is initiated by the reprogramming of cellular regulatory pathways that induce a coordinated downregulation of cell-cell adhesion and the acquisition of motility and invasiveness in a subpopulation of cancer cells. Epithelial-mesenchymal transition (EMT) has been proposed as a key regulatory program that drives these early metastasis-related changes ${ }^{[4]}$. The process of EMT can be induced by exposure of cancer cells to an altered microenvironment (e.g., hypoxia, pro-inflammatory cytokines), often in conjunction with somatic mutations ${ }^{[5,6]}$.

We previously reported that prolonged mammosphere culture induced EMT in two distinct epithelial breast cancer cell lines, MCF-7 and BT-474 cells, and generated stable populations of mesenchymal cancer cells, MCF-7M and BT- $474 \mathrm{M}^{[7,8]}$. EMT promoted a more glycolytic phenotype compared to the parental epithelial breast cancer cells that primarily use oxidative phosphorylation to produce ATP (submitted manuscript). The altered metabolic phenotype induced by EMT involved enhanced aerobic glycolysis (i.e., the Warburg effect) along with higher rates of lactate production and extracellular acidification.

As a result of the glycolytic shift induced by EMT, mesenchymal cells co-export higher amounts of lactate and protons to the extracellular microenvironment, thereby creating an acidic microenvironment that has been associated with tumor progression ${ }^{[0]}$. Elevated levels of lactate within the tumor microenvironment have been associated with metastasis and poor prognosis of cancer patients ${ }^{[10-12]}$. Once exported, lactate can act both as a carbon source for other cells and as a signaling molecule. For example, lactate produced by highly glycolytic cancer cells can be utilized by neighboring oxidative cancer cells for ATP production, a phenomenon known as "metabolic symbiosis" ${ }^{[13,14]}$. As a "signaling molecule", lactate can regulate migration, angiogenesis, immune escape and metastasis ${ }^{[15-17]}$.

Intracellular and extracellular lactate levels are determined, in part, by different isoforms of the lactate/H+ symporter, termed monocarboxylate transporters $(\mathrm{MCTs})^{[18,19]}$. MCT isoforms are lactate/proton co-transporters, and thus contribute to the acidification and lactate accumulation and signaling in the tumor microenvironment ${ }^{[20]}$. 
However, different isoforms have different affinities for lactate and pyruvate, which influences whether they act predominantly as cellular exporters or importers of lactate and H+. MCT1 is a ubiquitous lactate/H+ symporter that is involved in both import and export of lactate, whereas MCT4 is primarily involved in lactate/ $\mathrm{H}+$ export. Both MCT1 and MCT4 are differentially expressed in several cancers, including breast cancer ${ }^{[21]}$. Additional important players in lactate shuttling between cancer cells are the lactate dehydrogenases A and $\mathrm{B}$ (LDHA and LDHB) that catalyze the reversible conversion of pyruvate to lactate, alter the NAD+/NADH ratio and thus glycolytic flux, and have also been implicated in EMT and metastasis ${ }^{[22]}$.

Previous studies have identified the cell-surface G-protein-coupled receptor, GPR81, as an endogenous lactate receptor. GPR81 family of receptors consists of three highly homologous members, GPR109a, GPR109b and GPR81, which are regulated by the specific agonists, 3-hydroxybutyrate, 3-hydroxyoctanoate, and lactate, respectively ${ }^{[23]}$. GPR81 has been mainly studied in adipocytes, in which the receptor is coupled to Gi/q. GPR81 activation by lactate decreases cAMP production, which ultimately reduces lipolysis ${ }^{[24]}$. Recently GPR81 has been shown to be upregulated in several cancers (pancreas, colon, liver, breast, cervix) and its expression levels associated with tumor growth and metastasis ${ }^{[25-29]}$. In this current study, we examined GPR81 expression in the context of EMT in breast cancer. We provide evidence that GPR81 promotes lactate transport by MCT1 in hormone-positive breast cancer cells and its expression is crucial for their cell proliferation and survival.

\section{METHODS}

\section{Cell culture}

BT-474 and MCF-7 cell lines were obtained from the American Type Culture Collection (Manassas, VA). EMT in MCF-7 and BT-747 cells was induced using prolonged mammosphere culture method as reported previously ${ }^{[7,8]}$. BT-474 and MCF-7 and their corresponding EMT-derived BT-474-M and MCF-7-M cells were grown in DMEM/F-12 containing 10\% heat inactivated FBS (Gibco, Grand Island, NY) and $1 \times$ MycoZap $^{\text {TM }}$ Plus-CL antibiotic (Lonza, Walkersville, MD). BT-474 and MCF-7 cells were additionally supplemented with $1 \times$ Insulin-Transferrin-Selenium solution (Gibco, Grand Island, NY). All cells were incubated at $5 \% \mathrm{CO}_{2}$ and at $37^{\circ} \mathrm{C}$.

\section{3-dimensional culture}

Corning $^{\mathrm{TM}}$ Matrigel $^{\mathrm{TM}}$ GFR (Fisher Scientific) or Matrigel ${ }^{\mathrm{TM}}$ (Corning) was used as culture matrix following a modified version of the previously described protocol for 3-dimensional on-top assay ${ }^{[30]}$. Twenty-four-well plates were coated with $200 \mathrm{ul}$ of the mix of 1:1 Matrigel ${ }^{\mathrm{TM}} /$ Physiological modified medium supplemented with $17 \mu \mathrm{mol} / \mathrm{L}$ Insulin-Transferrin-Selenium solution (Gibco, Grand Island, NY) and kept at $37^{\circ} \mathrm{C}$ for 30 min to allow the Matrigel ${ }^{\mathrm{TM}}$ to solidify. All cell lines were suspended in physiological modified medium supplemented with $1 \times$ insulin and seeded into the coated twenty-four-plates. Epithelial and mesenchymal cells were seeded in $250 \mu \mathrm{L}$ at 100,000 cells/well and 20,000 cells/well, respectively, and incubated at $37^{\circ} \mathrm{C}$ for $30 \mathrm{~min}$ to allow cell attachment to the Matrigel. All wells were overlaid with $250 \mu \mathrm{L}$ of physiological modified medium containing $4 \% \mathrm{Matrigel}^{\mathrm{TM}}$. Culture medium was replaced with fresh medium daily and cells were used for each assay after seven days, unless otherwise indicated.

\section{Physiological modified medium and drugs}

RPMI 1640 Medium Modified without L-glutamine, amino acids and glucose (powder, R9010-02; US Biological, Swampscott, MA) was supplemented with the following: from Sigma (St. Louis, MO) - 1X MEM amino acid mixture (minus glutamine) (M5550), 1× Lipid Mixture 1 (L0288), $14.3 \mathrm{mmol} / \mathrm{L}$ sodium bicarbonate powder (S5761); from ThermoFisher/Gibco, (Waltham, MA) -1× non-essential amino acid mixture (11140), $10 \%$ heat-inactivated FBS (16000069) and $15 \mathrm{mmol} / \mathrm{L}$ HEPES (15630-106); from (Lonza, Walkersville, MD) - $1 \times$ MycoZap ${ }^{\mathrm{TM}}$ Plus-CL antibiotic. 
Unless otherwise noted, this medium was also supplemented with physiological levels of: glucose $(5 \mathrm{mmol} / \mathrm{L}$; (Gibco A24940), sodium pyruvate (0.1 mmol/L; Gibco 11360-070), Na-lactate (1 mmol/L; Sigma L7022) and L-glutamine (0.5 mmol/L; Sigma G7513. This same medium, but without phenol red (powder, R9010-01; US Biological, Swampscott, MA, was used in assays where cells were treated with $\beta$-Estradiol or Tamoxifen.

MCT1 inhibitor SR 13800, $\beta$-Estradiol and Tamoxifen (Tocris Bioscience, Bristol, UK) solutions were used at concentrations of $100 \mathrm{nmol} / \mathrm{L}, 10 \mathrm{nmol} / \mathrm{L}$ and $1 \mu \mathrm{mol} / \mathrm{L}$, respectively.

\section{Imaging of 3-dimensional structures}

Cells were cultured in 3-dimensional Matrigel and glass bottom 24-well plate (MatTek Corporation, MA) for seven days. Phase contrast images of the 3-dimensional structures of epithelial and mesenchymal cells were imaged using a Zeiss Axio Observer inverted microscope (Carl Zeiss MicroImaging Inc., Thomwood, NY) equipped a QImaging Retiga EXi CCD digital camera (QImaging, Surrey, BC). For immunofluorescence images cell lines grown in 3-dimensional Matrigel at the seventh day were fixed in $2 \%$ formaldehyde in $1 \times$ PBS for $20 \mathrm{~min}$. Cells were washed with $1 \times$ PBS and then treated with $0.1 \%$ Triton-X 100 for $10 \mathrm{~min}$. Cell were washed with $1 \times$ PBS and blocked with $1 \%$ bovine serum albumin (BSA) for 20 min. After blocking, cells were incubated with F-actin-staining solution for $20 \mathrm{~min}$ at room temperature. F-actin-staining solution was prepared with $5 \mu \mathrm{L}$ Alexa-488 Phallotoxin (Invitrogen, Carlsbad, CA) stock in $200 \mu \mathrm{L} 1 \mathrm{X}$ PBS for each well to be stained. SYTOX ${ }^{\mathrm{TM}}$ orange dye (Invitrogen, Carlsbad, CA) was used to stain nuclei. Images were captured using a Zeiss Pascal confocal system with a 40× 1.2 NA objective (Carl Zeiss Microscopy, Narashige, MN).

\section{Transient transfection of siRNA}

MCF-7 cells were seeded in $250 \mathrm{uL}$ at a density of 150, 000 cells/well in twenty-four-well plates coated with $200 \mu \mathrm{L}$ of the mix of 1:1 Matrigel ${ }^{\mathrm{TM}} /$ Physiological modified medium supplemented with $17 \mu \mathrm{mol} / \mathrm{L}$ Insulin-TransferrinSelenium solution (Gibco, Grand Island, NY) and transiently transfected with Trilencer-27 human siRNA (siNT and siGATA3 "A") at a final concentration of $10 \mathrm{nmol} / \mathrm{L}$ (Origene, Rockville, MD) with Lipofectamine ${ }^{\mathrm{TM}}$ RNAiMAX transfection reagent (Thermo Scientific, Rockford, IL). After $8 \mathrm{~h}$ of transfection cells were overlaid with $250 \mu \mathrm{L}$ of physiological modified medium containing $4 \%$ Matrigel $^{\mathrm{TM}}$. Culture medium was replaced with fresh medium every day and cells were used for each assay after 72 or $96 \mathrm{~h}$ after transfection.

\section{Growth and viability assays}

MCF-7 cells were seeded at a density of 150, 000 cells/well and recovered from Matrigel by incubating them with $5 \mathrm{mM}$ EDTA (Gibco, Grand Island, NY) in ice cold $1 \mathrm{X}$ PBS for 30 minutes at $4{ }^{\circ} \mathrm{C}$ and centrifuged for $5 \mathrm{~min}$ at $1000 \mathrm{rpm}$. Recovered cells were digested with $0.25 \%$ trypsin for $4 \mathrm{~min}$ and resuspended in DMEM with $10 \%$ FBS. The cell number was calculated on hemocytometer at specific time point. Viability assays were performed three times for each cell group.

\section{Real-time qPCR and primers}

Total RNA was isolated from cell lines using TRIzol Reagent (Ambion RNA, Carlsbad, CA), following the manufacturer's instruction. cDNA was synthesized $1 \mu \mathrm{g}$ of total RNA using iSCRIPT cDNA synthesis kit (Bio-Rad, Hercules, CA). SYBR green based SsoFast EvaGreen Supermix (Bio-Rad, Hercules, CA) was utilized to perform real-time PCR from $50 \mathrm{ng}$ of cDNA. Gene expression was normalized to TATA-box binding protein using $2^{-\Delta \mathrm{Ct}}$ method. Specificity of the primer sets was confirmed by melting curve analysis.

\section{cDNA arrays}

Real-time qPCR was performed in two TissueScan ${ }^{\mathrm{TM}}$ breast cancer and normal tissue cDNA arrays I and II (Origene, Rockville, MD). Gene expression was normalized to normal tissue using $2^{-\Delta \mathrm{Ct}}$ method. 


\section{Glucose uptake and lactate production}

Cells were cultured and transfected in 3-dimensional Matrigel ${ }^{\mathrm{TM}}$ with physiological modified medium for 72 or $96 \mathrm{~h}$ and medium was changed daily. Glucose and lactate concentrations in the culture medium were determined by fluorometric based Glucose Assay Kit and Lactate Assay Kit (BioVision, Inc., Milpitas, CA) according to the vendor's instructions using Synergy 2 Multi-Mode Microplate Reader (BioTek, Winooski, VT). The amount consumed or produced by cells was determined by comparing the concentration in the medium incubated without cells and then normalized to cell number. These assays were performed three times with two experimental replicates for each cell type.

\section{Western blot and antibodies}

Cells were collected form 3-dimensional Matrigel ${ }^{\mathrm{TM}}$ culture (as described previously) and lysed with RIPA buffer (TEKnova, Hollister, CA) containing Halt Protease and Phosphatase Inhibitor Cocktail (Thermo Scientific, Rockford, IL). The lysates were clarified by centrifugation at $4{ }^{\circ} \mathrm{C}$ for $10 \mathrm{~min}$ at $12,000 \times \mathrm{g}$. The concentration of protein in the supernatants was measured using BCA assay (Thermo Scientific Pierce, Rockford, IL). Total proteins ( $20 \mu \mathrm{g} / \mathrm{lane})$ were separated on a $10 \%$ SDS-PAGE and were transferred to nitrocellulose membrane. The membranes were blocked with 5\% BSA in $1 \times$ TBST ( $\beta$-actin) or with 5\% non-fat milk (MCT1, MCT4 and Cleaved PARP) and subsequently incubated with primary and secondary antibodies according to the vendor's instructions. Blots were visualized by using Amersham ECL prime (GE Healthcare Life Sciences, Buckinghamshire, UK) and imaged using G:box imaging system (SynGene, Cambridge, UK). Primary antibodies to Cleaved PARP (1:1000) was given by Dr. Claffey from Cell Signaling Technology (Danvers, MA); $\beta$-actin (1:2500) and MCT1 (1:1000) were purchased from Abcam (Cambridge, MA). MCT4 (1:1000) was purchased from Millipore (Billerica, MA). All images were imported to Image J for pixel grayscale intensity analysis. Western blot analysis was performed three times using two independent protein samples for each cell group.

\section{Nuclear magnetic resonance spectroscopy and data analysis}

Cells were collected from 3-dimensional Matrigel ${ }^{\mathrm{TM}}$ culture (as described previously) and lysed with 1:1:1 methanol/chloroform/water in sequence with $5 \mathrm{~s}$ of gyration between additions. In order to optimize chemical extraction, non-trypsin-based methods were employed as follows ${ }^{[31]}$. A $10 \mu \mathrm{L}$ sample was then removed for cell counting. Resulting lysed solution was then allowed to separate overnight at $4{ }^{\circ} \mathrm{C}$ to form resultant aqueous and organic layers. Aqueous layer containing metabolites of concern were then manually extracted and resulting solution was subjected to addition of Chelex (approximate $3 \mathrm{mg} / \mathrm{mL}$ ) for removal of divalent ions, which was then removed through vacuum filtration using Buchner filter. Solution was then lyophilized overnight to form solid components. $800 \mu \mathrm{L}$ of D2O with $10 \mathrm{mmol} / \mathrm{L}$ EDTA and $1 \mathrm{mmol} / \mathrm{L} \mathrm{4,} \mathrm{4-dimethyl-4-silapentane-1-sulfonic} \mathrm{acid} \mathrm{(DSS)} \mathrm{was} \mathrm{added} \mathrm{and} \mathrm{solution} \mathrm{was} \mathrm{centrifuged}$ at $3000 \mathrm{rpm}$ for $5 \mathrm{~min}$ prior to placement in standard $5 \mathrm{~mm}$ nuclear magnetic resonance (NMR) tube for $1 \mathrm{H}$ NMR.

1H NMR experiments were performed on $500 \mathrm{MHz}$ Agilent spectrometer with HCN cold probe and a VNMRS console. Default pre-saturation pulse sequences were utilized with the following parameters: pulsewidth $=7.2 \mu \mathrm{s}$, sweep-width $=8389.26 \mathrm{~Hz}, 2460$ complex points, $\mathrm{D} 1=1.5 \mathrm{~s}$, SATPWR $=6 \mathrm{~dB}$ on $\mathrm{H}_{2} \mathrm{O}$ signal, and 64 transients. Resulting $1 \mathrm{H}$ NMR underwent Fourier Transformation and data was further analyzed in MestReNova NMR software, version 9.0.0. Processing template for all spectra had the following time domain corrections: drift correction to tail points of $5 \%$ and apodization through an exponential of $1.5 \mathrm{~Hz}$. The frequency domain had global phase correction and a baseline correction using Bernstein polynomial of $n=3$. Reference signal was set as the singlet produced off of DSS at $0 \mathrm{ppm}$. Lactate and Pyruvate were identified on the spectra through prior titration analyses and the absolute area under the curve was used for their methyl groups with respect to methyl groups found off of DSS with the usage of Line-Shape analysis. These areas were used to calculate concentrations by a ratio of the absolute area to the number of protons 
A
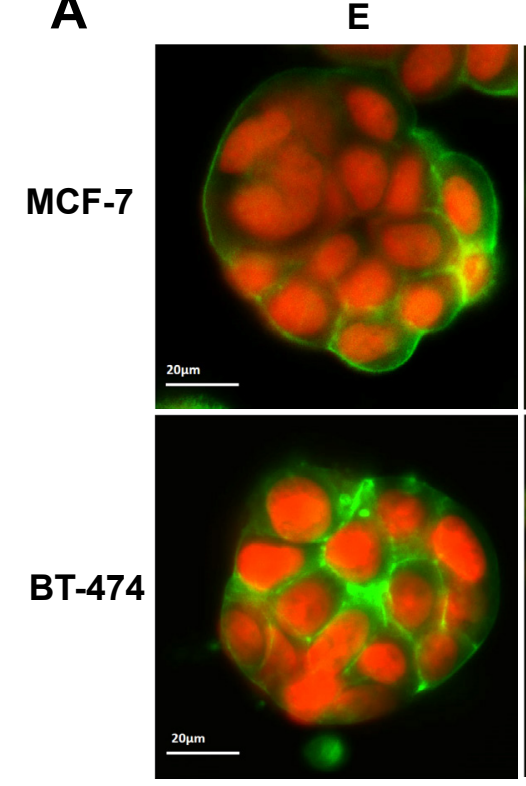

M

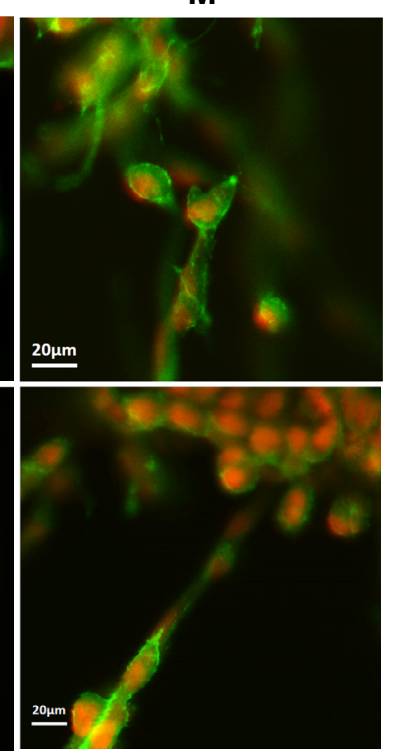

B

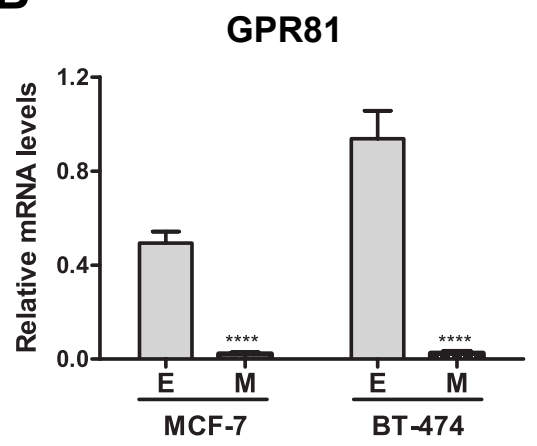

Figure 1. GPR81 expression of epithelial MCF-7 and BT-474 cells and mesenchymal MCF-7M and BT-474M in 3-dimensional Matrigel culture. A: fluorescence microscopy images of 3-dimensional structures of proliferating epithelial (E) and mesenchymal (M) cells in 3-dimensional Matrigel with physiological modified medium for 7 days. Green, Phalloidin staining of F-actin; red/orange, nuclei counterstained with SYTOXTM orange nucleic acid (red); B: real-time qPCR analysis of GPR81 in epithelial (E) and mesenchymal (M) cells. Results are presented as mean \pm S.E.M. ${ }^{\star} P<0.05 ;{ }^{\star \star} p<0.01$; ${ }^{\star \star \star} p<0.001 ;{ }^{\star \star \star \star} p<0.0001$ (ordinary one-way ANOVA)

in the signal used. Resulting concentrations in millimolar were then exported into an Excel spread sheet containing cellular counts to determine a normalized concentration per number of cells.

\section{Statistical analysis}

Ordinary one-way ANOVA followed by Sidak's multiple comparison post-test or two-way ANOVA was performed to determine statistical significance. $P<0.05$ was considered to be significant. Statistical analysis was performed using GraphPad Prism software (La Jolla, CA).

\section{RESULTS}

\section{Endogenous lactate receptor GPR81 is upregulated in epithelial breast cancer cells}

Epithelial (E) MCF-7 and BT-474 cell lines and the corresponding post-EMT mesenchymal (M) MCF-7M and BT-474M were cultured in a more physiologically relevant system using 3-dimensional Matrigel and physiological modified medium (referred as MPM culture). Under these conditions epithelial breast cancer cells grew as tightly adherent spheroids for 1 week [Figure 1A; "E" boxes]. The two mesenchymal cell lines also formed tight spheroids initially, but after 2 days began invading the Matrigel [Figure 1A; "M" boxes]. After 1 week of MPM culture, cells were examined for the expression of the endogenous lactate receptor GPR81. Epithelial spheroids expressed relatively high levels of GPR81, whereas mesenchymal cells showed no or very low expression [Figure $1 \mathrm{~B}]$.

\section{GPR81 is highly expressed in human hormone-receptor-positive breast cancer tissues}

To determine whether GPR81 was present in human breast cancer tumors, we analyzed mRNA gene expression profiles from 74 human breast cancer samples and 12 non-tumorigenic (NT) breast tissues [Figure $2 \mathrm{~A}$ ]. We grouped these samples in non-tumorigenic (NT), estrogen receptor positive $\left(\mathrm{ER}^{+}\right)$, amplification of the human epidermal receptor growth factor $2\left(\mathrm{HER}_{2}{ }^{+}\right)$and triple negative breast cancer (TNBC) according to their clinical information provided by the supplier. Higher levels of GPR81 mRNA were noted in hormone- 
A

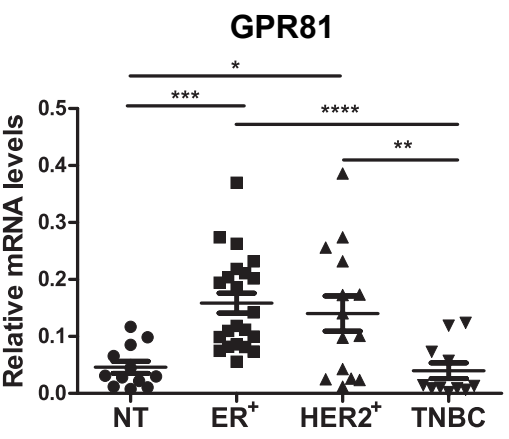

B

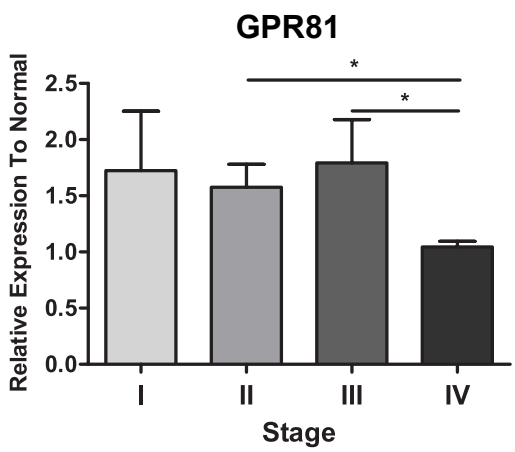

C Kaplan-Meier Survival Curve

With number of subjects at risk

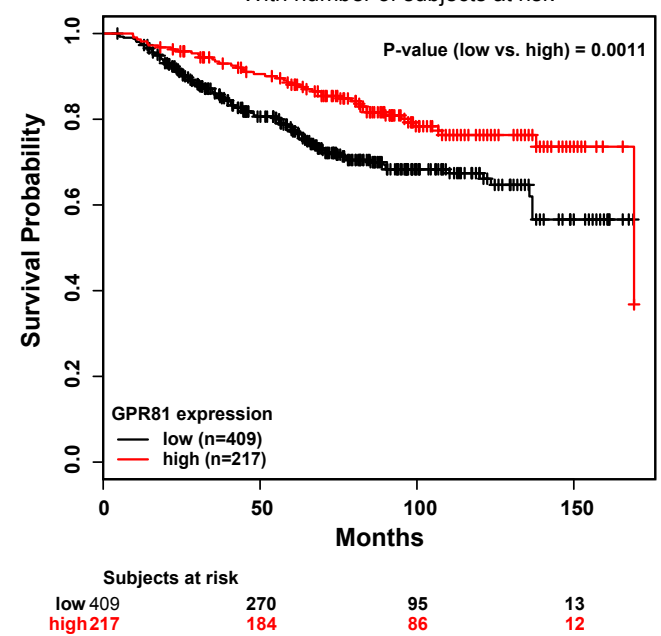

Figure 2. GPR81 is highly expressed in human hormone-receptor-positive breast cancer tissues. A: relative mRNA expression of GPR81 in $\mathrm{ER}^{+}(n=32), \mathrm{HER}^{+}(n=17)$ and TNBC $(n=12)$ breast cancer tissues to normal tissue $(n=12)$; B: relative mRNA expression of GPR81 in stage I $(n=21)$, II $(n=32)$, III $(n=25)$ and IV $(n=4)$ of breast cancer; C: Kaplan-Meier plot of estimated overall survival in breast cancer patients ( $n=626$ ) with low expression of GPR81, below the median (black line) or high expression of GPR81, above the median (red line). Log-rank test $P$-value $=0.0011$. Results are presented as mean \pm S.E.M. ${ }^{\star} P<0.05 ;{ }^{\star \star} P<0.01 ;{ }^{\star \star \star} P<0.001$ (two-way ANOVA)

positive breast cancer samples $\left(\mathrm{ER}^{+}\right.$and $\mathrm{HER} 2^{+}$) and significantly lower levels were found in the NT and TNBC samples [Figure 2A]. We also analyzed the relative gene expression of GPR81 by the stage of human breast cancer. Breast cancer stage II-III had significantly higher GPR81 expression than stage IV [Figure 2B]. Additionally, the Kaplan-Meir survival plot showed that high expression levels GPR81 was strongly associated with better prognosis and overall survival in breast cancer patients [Figure $2 \mathrm{C}](P=0.0011)(n=626)^{[32]}$. We also tested GPR81 expression in other breast cancer cell lines. Hormone-receptor positive HC1500 cells had higher expression of the lactate receptor and lactate importer compared to the TNBC, MDA-MB231 cells [Supplementary Figure 1A-C]. These results demonstrate that GPR81 is mainly expressed in hormone positive and less aggressive types of human breast cancer.

\section{GPR81 regulates expression of the gene involved in lactate import in MCF-7 epithelial breast cancer cell line}

A previous study reported that GPR81 regulates the expression of lactate transport related genes MCT1 and MCT4 in pancreatic cancer cells through an as yet unknown mechanism ${ }^{[26]}$. To determine the role of the endogenous lactate receptor GPR81 in the lactate metabolism of estrogen positive breast cancer cells, we silenced GPR81 ( 60\%) in MCF-7 cancer cells using siRNA (siGPR81) up to four days in MPM culture [Figure 3A]. GPR81 knockdown in MCF-7 cells led to a 2-fold reduction mRNA and protein expression of the lactate importer, MCT1, but had no effect on the lactate exporter, MCT4 [Figure 3B and C]. In addition, GPR81 did not significantly alter the expression of other lactate metabolism-related genes (LDHA, LDHB, MCT2) [Supplementary Figure 2].

\section{GPR81 regulates lactate uptake in MCF-7 epithelial breast cancer cell line}

We found that the two epithelial breast cancer cell lines had higher steady state levels of intracellular lactate and pyruvate than the corresponding post-EMT mesenchymal cell lines ${ }^{[33]}$. Given that MCT1 is primarily a 
A

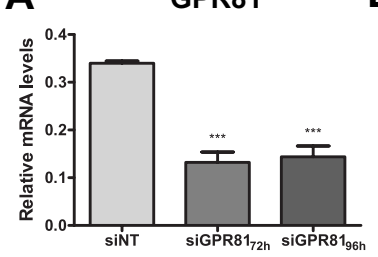

B

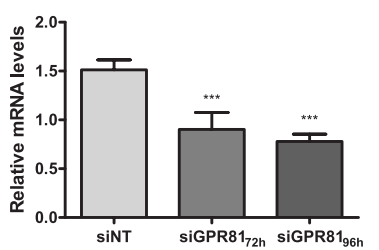

MCT4

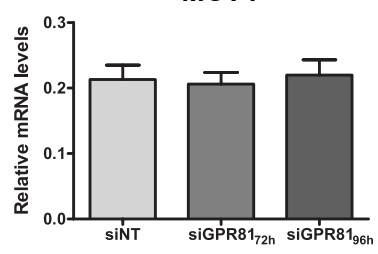

C

MCT1

MCT4

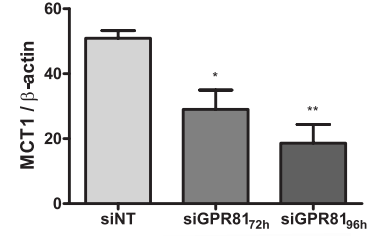

MCT1

$\beta$-actin
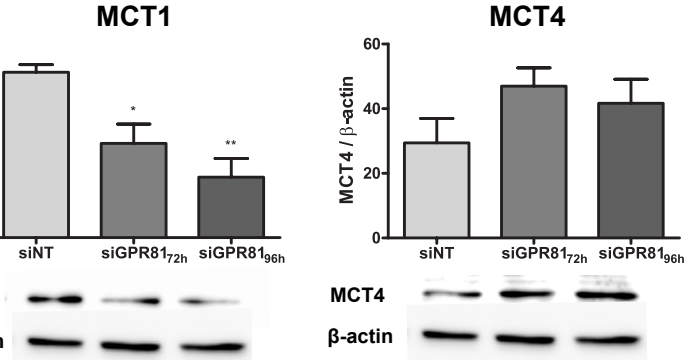

Figure 3. GPR81 regulates expression of the gene involved in lactate import in MCF-7 epithelial breast cancer cell line. A: real-time $\mathrm{QPCR}$ analysis of GPR81; B: MCT1 and MCT4 mRNA levels in MCF-7-siNT (control) and MCF-7-siGPR81 cultured in 3-dimensional Matrigel with physiological modified medium at 72 and $96 \mathrm{~h}$ after transfection; C: Western blot analysis of cell lysates from MCF-7-siNT and MCF7-siGPR81 cells used to detect protein expression levels of MCT1 and MCT4. $\beta$-actin was used as a loading control. The bars represent the mean \pm S.E.M of three independent experiments; ${ }^{\star} P<0.05$; ${ }^{\star \star} P<0.01 ;{ }^{\star \star \star} P<0.001$; ${ }^{\star \star \star \star} P<0.0001$ (ordinary one-way ANOVA)

A

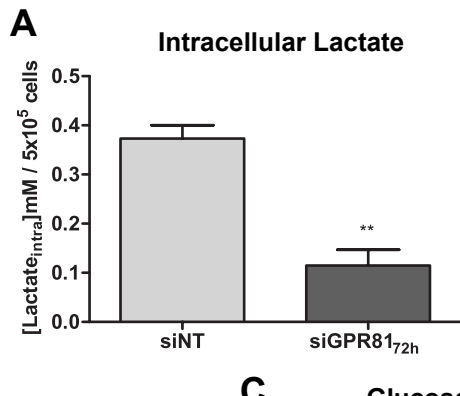

B

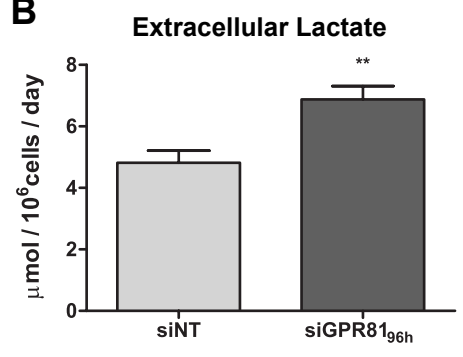

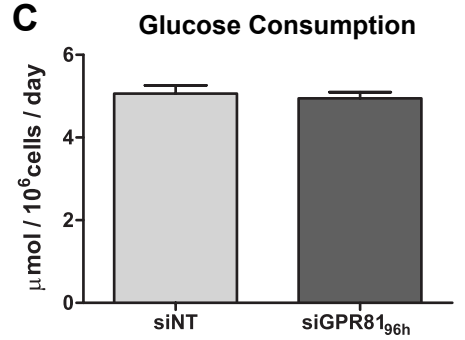

Figure 4. GPR81 regulates lactate uptake in MCF-7 epithelial breast cancer cell lines. A: intracellular lactate of MCF-7-siNT (control) and MCF-7-siGPR81 measured using NMR analysis after $72 \mathrm{~h}$ of transfection; B: lactate concentration in the medium of MCF-7-siNT $10 \mathrm{nmol} / \mathrm{L}$ and MCF-7-siGPR81 $10 \mathrm{nmol} / \mathrm{L}$ were measured after $96 \mathrm{~h}$ of transfection; C: glucose consumption rate of MCF-7-siNT and MCF-7- siGPR81 were measured after $96 \mathrm{~h}$ of transfection. MCF-7-siNT and MCF-7-siGPR81 cells were cultured and transfected in 3-dimensional Matrigel with physiological modified medium and measurements were taken after $24 \mathrm{~h}$ of culture in fresh medium. The bars represent the mean \pm S.E.M of three independent experiments; ${ }^{\star} P<0.05 ;{ }^{\star \star} P<0.01$; (ordinary one-way ANOVA)

lactate importer, we hypothesized that the reduction of MCT1 expression may affect the lactate uptake of the epithelial MCF-7 breast cancer cells. To test this hypothesis, we measured the intracellular lactate of nontargeted control MCF-7-siNT and MCF-7-siGPR81 in MPM culture using NMR analysis. MCF-7-siGPR81 cells had significant lower intracellular lactate compared to MCF-7-siNT cells [Figure 4A], indicating reduced lactate uptake of MCF-7-siGPR81 cells. Additionally, GPR81 knockdown resulted in higher levels of lactate 
A

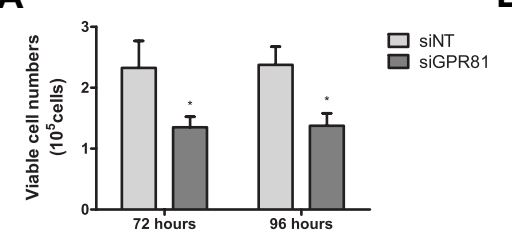

C
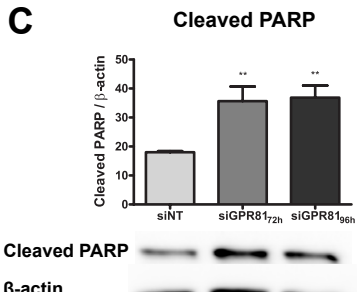

D

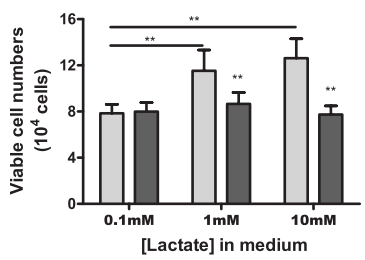

B
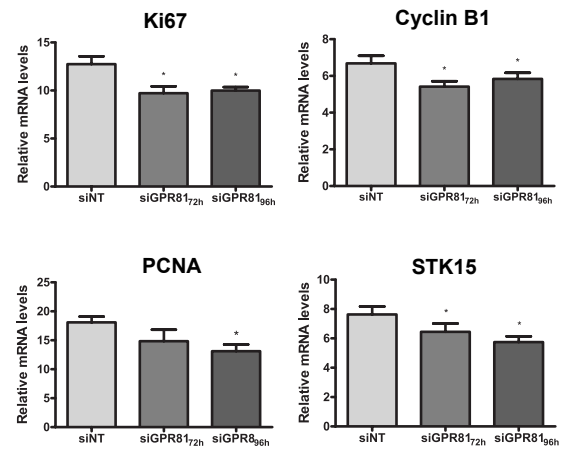

Figure 5. GPR81 is required for cancer cell proliferation and cancer cell survival when lactate is the primary fuel source. A: viable cell counts of MCF-7-siNT (control) and MCF-7-siGPR81 after 72 and $96 \mathrm{~h}$ of transfection and subtracting the initial cell number; B: real-time qPCR analysis of proliferation markers: Ki67, Cyclin B1, PCNA and STK15; C: Western blot analysis of cell lysates from MCF-7-siNT and MCF7 -siGPR81 cells after 72 and $96 \mathrm{~h}$ of transfection used to detect protein expression levels of the apoptotic maker cleaved-PARP. $\beta$-actin was used as a loading control; D: MCF-7-siNT and MCF-7-siGPR81 breast cancer cells were cultured in 3-dimensional Matrigel with physiological modified medium containing only lactate at $0.1 \mathrm{mmol} / \mathrm{L}, 1 \mathrm{mmol} / \mathrm{L}$ and $10 \mathrm{mmol} / \mathrm{L}$ concentration for $96 \mathrm{~h}$; E: MCF-7 breast cancer cells were cultured in 3-dimensional Matrigel with physiological modified medium containing only lactate at $0.1 \mathrm{mmol} / \mathrm{L}, 1 \mathrm{mmol} / \mathrm{L}$ and $10 \mathrm{mmol} / \mathrm{L}$ concentration and treated with or without MCT1 inhibitor SR 13800 for $96 \mathrm{~h}$. Viable cell counts after treatment. Results are presented as mean \pm S.E.M. of three independent experiments; ${ }^{\star} P<0.05 ;{ }^{\star \star} P<0.01 ;{ }^{\star \star \star} P<0.001$ (ordinary one-way ANOVA)

in the culture medium but not control MCF-7-siNT cells [Figure 4B]. We did not detect significant change in glucose consumption [Figure $4 \mathrm{C}$ ]. Taken together, these data suggest the role for GPR81 in the regulation of the lactate uptake of the epithelial MCF-7 breast cancer cells.

\section{GPR81 is required for cancer cell proliferation and cancer cell survival when lactate is the primary}

\section{fuel source}

Lactate has been previously suggested as an alternative energy source for aerobic breast cancer cells ${ }^{[13,14,34]}$. To determine whether GPR81 plays a role in breast cancer cell proliferation, we studied the increase in viable cell numbers and the expression of cell proliferation makers (taken from the OncotypeDx recurrence score assay) in control MCF-7-siNT and MCF-7-siGPR81 cells. Silencing of GPR81 led to about 40\% reduction in cell proliferation of MCF-7-siGPR81 [Figure 5A] and decreased relative gene expression of proliferation makers; Ki67, Cyclin B1, PCNA and STK15 [Figure 5B]. Furthermore, the expression of the cell apoptosis marker, cleaved PARP, was significantly increased by 2-fold in MCF-7-siGPR81 cells when compared to control MCF-7-siNT cells [Figure 5C].

To determine whether GPR81 expression is required for cell growth and survival, we cultured control MCF-siNT and MCF-7-siGPR81 cells in 3-dimensional Matrigel with physiological medium lacking glucose, glutamine and pyruvate and with $0.1 \mathrm{mmol} / \mathrm{L}, 1 \mathrm{mmol} / \mathrm{L}$ or $10 \mathrm{mmol} / \mathrm{L}$ lactate as the main available nutrient source. Despite of the significant increase in cell number of the MCF-7-siNT cells when cultured in medium containing only $1 \mathrm{mmol} / \mathrm{L}$ and $10 \mathrm{mmol} / \mathrm{L}$ lactate, GPR81 silencing prevented cell growth under these nutrient-limited 
A

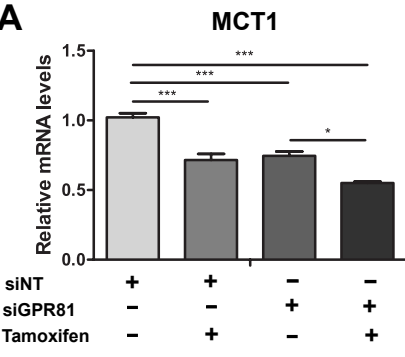

B

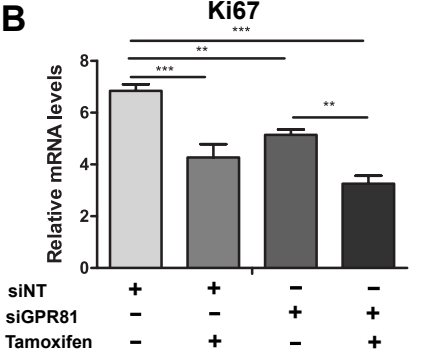

C

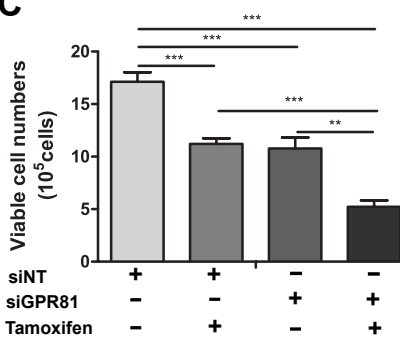

PR

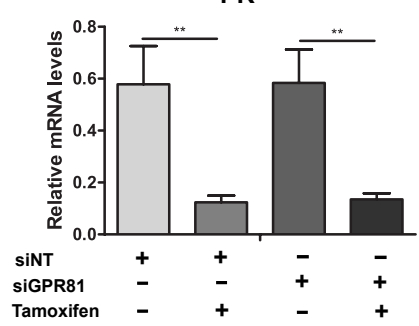

CyclinB1
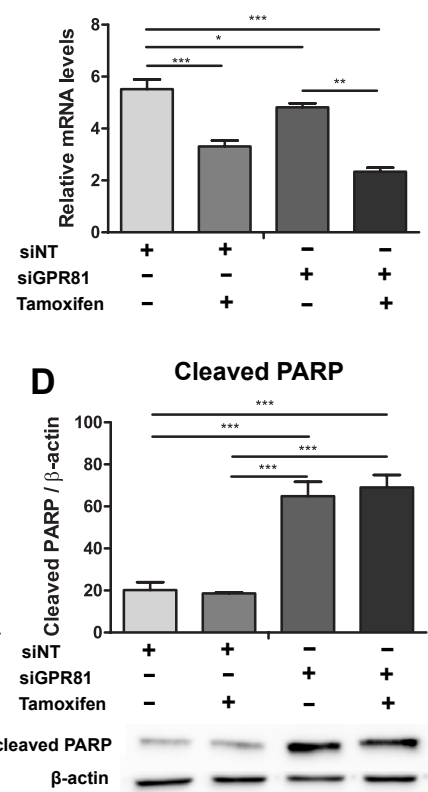

Figure 6. Additive effect of GPR81 knockdown and Tamoxifen treatment in reducing the cell proliferation and increasing cell apoptosis in epithelial MCF-7 breast cancer cells. MCF-7-siNT (control) and MCF-7-siGPR81 cancer cells were treated with or without $1 \mu \mathrm{mol} / \mathrm{L}$ Tamoxifen for $96 \mathrm{~h}$ in 3-dimensional Matrigel with physiological modified medium. A: real-time qPCR analysis of lactate importer MCT1; and progesterone receptor PR; B: relative mRNA expression of proliferation markers: Ki67 and Cyclin B1; C: viable cell counts of epithelial MCF-7-siNT and MCF-7-siGPR81; D: Western blot analysis of cell lysates from MCF-7-siNT and MCF-7-siGPR81 used to detect protein expression levels of the apoptotic maker cleaved-PARP. $\beta$-actin was used as a loading control. Results are presented as mean \pm S.E.M. of three independent experiments ${ }^{\star} P<0.05 ;{ }^{\star \star} P<0.01 ;{ }^{* \star \star} P<0.001 ;{ }^{\star \star \star \star} P<0.0001$ (ordinary one-way ANOVA)

conditions [Figure 5D]. Similarly, we found that treatment with the MCT1 inhibitor also prevented cell growth when in MCF-7 cells were culture in nutrient-limited conditions [Figure 5E]. Taken together, these data support the physiological role for GPR81 in the regulation of lactate uptake, cell proliferation and survival of the epithelial MCF-7 breast cancer cells under nutrient-limited conditions.

\section{Tamoxifen treatment in reducing cell proliferation and increasing cell apoptosis has additive effect on GPR81 knockdown}

We observed here that $1 \mu \mathrm{mol} / \mathrm{L}$ Tamoxifen further reduced MCT1 gene expression in GPR81-silenced MCF7 cells treated for 4 days [Figure 6A]. The effect of Tamoxifen was confirmed by the reduced expression of the progesterone receptor ${ }^{[35]}$. We next evaluated the effects of Tamoxifen and GPR81 silencing in cell proliferation and apoptosis in ER+ MCF-7 breast cancer cells. GPR81 knockdown and Tamoxifen treatment had an additive effect on the reduction of cell proliferation markers; Ki67 and Cyclin B1 [Figure 6B], as well as viable cell numbers of MCF-7 cells in MPM culture [Figure 6C]. Additionally, cell apoptosis was significantly increased only in MCF-7-siGPR81 cells independently of Tamoxifen treatment, showed by the increased expression of the apoptotic marker cleaved PARP [Figure 6E]. These data suggest GPR81 regulates cell proliferation and apoptosis. 


\section{DISCUSSION}

The metabolic changes that cancer cells undergo in order to support macromolecule biosynthesis, growth and survival, are collectively referred to as "metabolic reprogramming", now considered a hallmark of cancer cell biolog $y^{[36,37]}$. Increasing evidence has linked metabolic reprogramming to metastatic transformation. For example, it has been shown that the EMT-transcription factor SNAIL mediates the suppression of mitochondrial respiration and enhances glycolysis in breast cancer ${ }^{[38,39]}$. In the accompanying report in this issue, we demonstrated that EMT induced a metabolic shift to a less oxidative and more glycolytic metabolism in two in epithelial cell lines MCF-7 and BT-474, as evidenced by increased glucose uptake and lactate production along with increased expression of enzymes and transporters. These studies suggest that metastatic transformation or EMT facilitates the metabolic swift toward glycolysis in some forms of breast cancer.

We also observed that metabolic reprogramming induced by EMT was associated with a striking switch in the expression of the lactate transporters MCT1 and MCT4. The two epithelial breast cancer cell lines expressed high levels of MCT1 and imported lactate as an alternative energy source. In contrast, the mesenchymal cells showed a marked decreased expression of MCT1 and upregulation of the lactate exporter MCT4. Our findings are consistent with those of Hussein and Brooks ${ }^{[40]}$, who reported MCT1 was expressed in MCF7 cells, but not in mesenchymal, triple-negative MDA-MB-231 cells, that were maintained in 2-dimensional culture containing high glucose medium. Similarly, MCT4 expression was lower in MCF7 cells as compared to the MDA-MB-231 cells. Using 2-dimensional culture and DMEM/F12 media with high glucose levels, Baenke et al. ${ }^{[41]}$ examined MCT4 expression and function in 17 breast cancer cell lines. Although their findings differed from ours in that MCT4 expression was highest in HER2-positive cell lines, these authors reported that MCT4 suppression increased mitochondrial oxidation, linking MCT4 to a more glycolytic phenotype. This is consistent with MCT4 promoting a glycolytic phenotype, and also raises the prospect that breast cancer cells can adapt to targeted therapies in part through metabolic reprogramming. In contrast to our finding of reciprocal expression between MCT1 and MCT4, Pinheiro et al.$^{[42]}$ used immunohistochemistry to analyze primary breast carcinomas for MCT1 and $\mathrm{MCT} 4$, and found MCT1 expression occurring most frequently in the Basal-like subtype and in higher grades of breast cancer. It is also important to note that expression of MCTs does not always correlate to lactate $/ \mathrm{H}^{+}$transport. For example, hypoxia-induced carbonic anhydrase IX enhanced the activity of MCT1 to export lactate and $\mathrm{H}^{+}$in MCF7 cells in 2-dimensional culture with physiological ( $5 \mathrm{mmol} / \mathrm{L}$ ) glucose levels. MCT4 upregulation was previously reported in triple negative breast cancer and high levels of this lactate exporter were associated with poor prognosis and survival ${ }^{[1,43]}$.

Lactate has been identified as the ligand for the endogenous cell-surface G-protein-coupled receptor 81 (GPR81). GPR81 was first discovered and mostly studied in adipocytes, in which lactate activates GPR81 to decrease the cAMP production to ultimately reduce lipolysis ${ }^{[24]}$. GPR81 is highly expressed in neurons ${ }^{[44]}$, and when activated, it is able to modify electrical activity of primary neuronal cells ${ }^{[45]}$. Also, the activation of GPR81 by lactate has been identified as the initial step for an anti-inflammatory response in pancreas, liver and uterus ${ }^{[46-48]}$.

More recently, GPR81 expression has been found to be upregulated in several types of cancer, including pancreatic, colon, liver, breast, lung and cervical cancers, and in several cases high GPR81 expression was associated with tumor growth, chemoresistance and metastasis ${ }^{[25-28]}$. It should also be noted that previous studies indicate that lactate activates GPR81 in the millimolar range (1-5 mmol/L), and in human breast cancer tissues lactate was found to be at concentrations as high as $8 \mathrm{mmol} / \mathrm{L}^{[14]}$, suggesting that GPR81 and downstream signaling pathways could be constantly activated by lactate in the breast tumor microenvironment. Our study provides the first examination of GPR81 expression in the context of EMT, and indicates that GPR81 expression is dependent on the type of breast cancer cell. We showed that hormone-positive epithelial breast cancer cells highly expressed GPR81. In contrast, EMT induction and 
transformation of epithelial breast cancer cells into triple negative mesenchymal cancer cells was associated with significantly reduced expression of GPR81. Lee et al. ${ }^{[25]}$ reported that GPR81 expression is significantly increased in breast cancer patients compared to normal mammary tissues. We also observed a significant association between GPR81 overexpression with ER $\alpha$-positive and human epidermal growth HER2 positive human breast cancer tissues as opposed to triple negative breast cancer. This is consistent with previous observations that ER $\alpha$-positive breast cancer tissues overexpressed GPR81 ${ }^{[25]}$. In addition, GPR81 was expressed at higher levels in the first three stages of breast cancer (Stage I, II and III) as compared to Stage IV, and GPR81 expression correlated with better overall survival of breast cancer patients. These findings suggest that GPR81 may be an important regulator in hormone-positive breast cancer and could be used as a prognostic marker in the progression of breast cancer.

GPR81 was reported to regulate the expression of genes involved in lactate metabolism, including lactate transporters, MCT1 and MCT4, in pancreatic cancer cells ${ }^{[26]}$. In partial agreement, our study revealed that GPR81 specifically regulates the lactate importer MCT1, but not lactate exporter MCT4, in epithelial breast cancer cells. Oxidative breast tumor cells with high expression of the lactate importer, MCT1, have been reported to import and oxidize extracellular lactate, a mechanism that is essential for cell viability under glucose deprivation ${ }^{[14,34,49]}$. In fact, Lee et al ${ }^{[25]}$ showed that epithelial breast cancer cells imported and utilized 14C-lactate for mitochondrial respiration. We previously showed that inhibition of MCT1 reduced cell proliferation in epithelial but not mesenchymal cancer cells when lactate was used as the primary extracellular fuel source. Here we report that GPR81 silencing caused downregulation of MCT1 and reduced cell growth in epithelial breast cancer cells grown in complete MPM medium. We reported that the two epithelial breast cancer cell lines contained significantly higher intracellular lactate and pyruvate that the two corresponding post-EMT mesenchymal cell lines (submitted manuscript). In the present study, we observed that silencing of GPR81 in MCF7 cells led to lower intracellular lactate and higher levels of lactate in the culture media, indicating reduction in lactate uptake. Furthermore, we found a protective effect of GPR81 against apoptosis, which was previously described in epithelial MCF-7 breast cancer cells, in which GPR81 activation triggered the PI3K/Akt signaling pathway to inhibit apoptosis ${ }^{[25]}$. Together these results suggest a specific regulation of GPR81 on lactate importer MCT1, which affects cell proliferation, apoptosis and survival of hormone-positive epithelial breast cancer cells.

In adipocytes, GPR81 is coupled to Gi/q which inhibits adenylate cyclase activity and decreases the production of cAMP. However, a previous study did not find a significant change in cAMP levels when GPR81 was silenced in epithelial MCF-7 breast cancer cells ${ }^{[25]}$, suggesting that GPR81 regulates MCT1 expression by another signaling pathway in this cells. Alternate GPR81-associated signaling that is independent of the PKA/cAMP pathway, but involves the GPR81-binding protein $\beta$-arrestin, has been reported in monocyte/ macrophage ${ }^{[48]}$. Further studies are needed to examine whether the $\beta$-arrestin pathway or other pathways are linked to GPR81 signaling in epithelial breast cancer, as these pathways may provide targets for future development of adjuvant therapies for Luminal A breast cancer.

\section{DECLARATIONS}

\section{Acknowledgments}

We thank Dr. Kevin Claffey, Department of Cell Biology, University of Connecticut for providing us the Cleaved PARP antibody and the cell line HC1500. This work supported by the Fund for Biology from the Cell Biology Department at the University of Connecticut Health Center.

\section{Authors' contributions}

Made substantial contributions to conception and design of the study and performed data analysis and interpretation: Tafur D, White B

Performed NMR studies, data acquisition, analysis and interpretation: Svrcek P 


\section{Availability of data and materials}

Not applicable.

\section{Financial support and sponsorship}

None.

\section{Conflicts of interest}

All authors declared that there are no conflicts of interest.

\section{Ethical approval and consent to participate}

Not applicable.

\section{Consent for publication}

Not applicable.

\section{Copyright}

(C) The Author(s) 2019.

\section{REFERENCES}

1. Torre LA, Bray F, Siegel RL, Ferlay J, Lortet-tieulent J, et al. Global cancer statistics, 2012. CA a cancer J Clin 2015;65:87-108.

2. Siegel RL, Miller KD, Jemal A. Cancer statistics. CA Cancer J Clin 2016;66:7-30.

3. Munzone E, Colleoni M. Optimal management of luminal breast cancer: how much endocrine therapy is long enough? Ther Adv Med Oncol 2018;10:175883591877743.

4. Nieto MA, Huang RYJ, Jackson RA, Thiery JP. EMT: 2016. Cell 2016;166:21-45.

5. Singh M, Yelle N, Venugopal C, Singh SK. EMT: mechanisms and therapeutic implications. Pharmacol Ther 2018;182:80-94.

6. Sarrió D, Rodriguez-Pinilla SM, Hardisson D, Cano A, Moreno-Bueno G, et al. Epithelial-mesenchymal transition in breast cancer relates to the basal-like phenotype. Cancer Res 2008;68:989-97.

7. Kondaveeti Y, Guttilla Reed IK, White BA. Epithelial-mesenchymal transition induces similar metabolic alterations in two independent breast cancer cell lines. Cancer Lett 2015;364:44-58.

8. Guttilla IK, Phoenix KN, Hong X, Tirnauer JS, Claffey KP, et al. Prolonged mammosphere culture of MCF-7 cells induces an EMT and repression of the estrogen receptor by microRNAs. Breast Cancer Res Treat 2012;132:75-85.

9. Kato Y, Ozawa S, Miyamoto C, Maehata Y, Suzuki A, et al. Acidic extracellular microenvironment and cancer. Cancer Cell Int 2013;13:89.

10. Ziebart T, Walenta S, Kunkel M, Reichert TE, Wagner W, et al. Metabolic and proteomic differentials in head and neck squamous cell carcinomas and normal gingival tissue. J Cancer Res Clin Oncol 2011;137:193-9.

11. Walenta S, Wetterling M, Lehrke M, Schwickert G, Sundfør K, et al. High lactate levels predict likelihood of metastases , tumor recurrence, and restricted patient survival in human cervical cancers. Cancer Res 2000;60:916-21.

12. Park J, Wysocki RW, Amoozgar Z, Maiorino L, Fein MR, et al. Cancer cells induce metastasis-supporting neutrophil extracellular DNA traps. Sci Transl Med 2016;8:361ra138.

13. Sonveaux P, Végran F, Schroeder T, Wergin MC, Verrax J, et al. Targeting lactate-fueled respiration selectively kills hypoxic tumor cells in mice. J Clin Invest 2008;118:3930-42.

14. Kennedy KM, Scarbrough PM, Ribeiro A, Richardson R, Yuan H, et al. Catabolism of exogenous lactate reveals it as a legitimate metabolic substrate in breast cancer. PLoS One 2013;8:e75154.

15. Choi SYC, Collins CC, Gout PW, Wang Y. Cancer-generated lactic acid: a regulatory, immunosuppressive metabolite? J Pathol 2013;230:350-5.

16. San-Millán I, Brooks GA. Reexamining cancer metabolism: lactate production for carcinogenesis could be the purpose and explanation of the Warburg Effect. Carcinogenesis 2017;38:119-33.

17. Sun S, Li H, Chen J, Qian Q. Lactic acid: no longer an inert and end-product of glycolysis. Physiology 2017;32:453-63.

18. Halestrap AP. The monocarboxylate transporter family-structure and functional characterization. IUBMB Life 2012;64:1-9.

19. Marchiq I, Pouysségur J. Hypoxia, cancer metabolism and the therapeutic benefit of targeting lactate/H(+) symporters. J Mol Med (Berl) 2016;94:155-71.

20. Morais-Santos F, Granja S, Miranda-Gonçalves V, et al. Targeting lactate transport suppresses in vivo breast tumour growth. Oncotarget 2015;6:19177-89.

21. Pinheiro C, Longatto-Filho A, Azevedo-Silva J, Casal M, Schmitt FC, et al. Role of monocarboxylate transporters in human cancers: state of the art. J Bioenerg Biomembr 2012;44:127-39. 
22. Zhang Y, Lin S, Chen Y, Yang F, Liu S. LDH-A promotes epithelial-mesenchymal transition by upregulating ZEB2 in intestinal-type gastric cancer. Onco Targets Ther 2018;11:2363-73.

23. Liu C, Wu J, Zhu J, Kuei C, Yu J, et al. Lactate inhibits lipolysis in fat cells through activation of an orphan G-protein-coupled receptor, GPR81. J Biol Chem 2009;284:2811-22.

24. Ahmed K, Tunaru S, Tang C, Müller M, Gille A, et al. An autocrine lactate loop mediates insulin-dependent inhibition of lipolysis through GPR81. Cell Metab 2010;11:311-9.

25. Lee YJ, Shin KJ, Park SA, Park KS, Park S, et al. G-protein-coupled receptor 81 promotes a malignant phenotype in breast cancer through angiogenic factor secretion. Oncotarget 2016;7:70898-911.

26. Roland CL, Arumugam T, Deng D, Liu SH, Philip B, et al. Cell surface lactate receptor GPR81 is crucial for cancer cell survival. Cancer Res 2014;74:5301-10.

27. Wagner W, Kania KD, Ciszewski WM. Stimulation of lactate receptor (HCAR1) affects cellular DNA repair capacity. DNA Repair (Amst) 2017;52:49-58.

28. Wagner W, Ciszewski WM, Kania KD. L- and D-lactate enhance DNA repair and modulate the resistance of cervical carcinoma cells to anticancer drugs via histone deacetylase inhibition and hydroxycarboxylic acid receptor 1 activation. Cell Commun Signal 2015;13:36.

29. Stäubert C, Broom OJ, Nordström A. Hydroxycarboxylic acid receptors are essential for breast cancer cells to control their lipid/fatty acid metabolism. Oncotarget 2015;6:19706-20.

30. Lee GY, Kenny PA, Lee EH, Bissell MJ. Three-dimensional culture models of normal and malignant breast epithelial cells. Nat Methods 2007:4:359-65.

31. Bi H, Krausz KW, Manna SK, Li F, Johnson CH, et al. Optimization of harvesting, extraction, and analytical protocols for UPLC-ESIMS-based metabolomic analysis of adherent mammalian cancer cells. Anal Bioanal Chem 2013;405:5279-89.

32. Lánczky A, Nagy Á, Bottai G, Munkácsy G, Szabó A, et al. MiRpower: a web-tool to validate survival-associated miRNAs utilizing expression data from 2178 breast cancer patients. Breast Cancer Res Treat 2016;160:439-46.

33. Tafur D, Svrcek P, Kondaveeti Y, Mehlmann L, Hoch J, et al. Reprogramming of Lactate Metabolism Associated With EpithelialMesenchymal Transition In Human Breast Cancer Cells. J Cancer Metastasis Treat. Forthcoming 2019.

34. Park S, Chang CY, Safi R, Liu X, Baldi R, et al. ERR $\alpha$-regulated lactate metabolism contributes to resistance to targeted therapies in breast cancer. Cell Rep 2016;15:323-35.

35. Allegra JC, Korat O, Do HM, Lippman M. The regulation of progesterone receptor by 17 beta estradiol and tamoxifen in the $\mathrm{Zr}-75-1$ human breast cancer cell line in defined medium. J Recept Res 1981;2:17-27.

36. Hanahan D, Weinberg RA. Hallmarks of cancer: the next generation. Cell 2011;144:646-74.

37. Pavlova NN, Thompson CB. The emerging hallmarks of cancer metabolism. Cell Metab 2016;23:27-47.

38. Lee SY, Jeon HM, Ju MK, Kim CH, Yoon G, et al. Wnt/snail signaling regulates cytochrome c oxidase and glucose metabolism. Cancer Res 2012;72:3607-17.

39. Dong C, Yuan T, Wu Y, Wang Y, Fan TW, et al. Loss of FBP1 by Snail-mediated repression provides metabolic advantages in basal-like breast cancer. Cancer Cell 2013;23:316-31.

40. Hussien R, Brooks GA. Mitochondrial and plasma membrane lactate transporter and lactate dehydrogenase isoform expression in breast cancer cell lines. Physiol Genomics 2011;43:255-64.

41. Baenke F, Dubuis S, Brault C, Weigelt B, Dankworth B, et al. Functional screening identifies MCT4 as a key regulator of breast cancer cell metabolism and survival. J Pathol 2015;237:152-65.

42. Pinheiro C, Albergaria A, Paredes J, Sousa B, Dufloth R, et al. Monocarboxylate transporter 1 is up-regulated in basal-like breast carcinoma. Histopathology 2010;56:860-7.

43. Doyen J, Trastour C, Ettore F, Peyrottes I, Toussant N, et al. Expression of the hypoxia-inducible monocarboxylate transporter MCT4 is increased in triple negative breast cancer and correlates independently with clinical outcome. Biochem Biophys Res Commun 2014;451:54-61.

44. Lauritzen KH, Morland C, Puchades M, Holm-Hansen S, Hagelin EM, et al. Lactate receptor sites link neurotransmission, neurovascular coupling, and brain energy metabolism. Cereb Cortex 2014;24:2784-95.

45. Bozzo L, Puyal J, Chatton JY. Lactate modulates the activity of primary cortical neurons through a receptor-mediated pathway. PLoS One 2013;8:e71721.

46. Lerch MM, Conwell DL, Mayerle J. The anti-inflammasome effect of lactate and the lactate GPR81-receptor in pancreatic and liver inflammation. Gastroenterology 2014;146:1602-5.

47. Madaan A, Nadeau-Vallée M, Rivera JC, Obari D, Hou X, et al. Lactate produced during labor modulates uterine inflammation via GPR81 (HCA1). Am J Obstet Gynecol 2017;216:60.e1-60.e17.

48. Hoque R, Farooq A, Ghani A, Gorelick F, Mehal WZ. Lactate reduces liver and pancreatic injury in toll-like receptor- and inflammasomemediated inflammation via gpr81-mediated suppression of innate immunity. Gastroenterology 2014;146:1763-74.

49. Boidot R, Veǵran F, Meulle A, Le Breton A, Dessy C, et al. Regulation of monocarboxylate transporter MCT1 expression by p53 mediates inward and outward lactate fluxes in tumors. Cancer Res 2012;72:939-48. 